\title{
Molecular analysis of polymorphic species of the genus Marshallagia (Nematoda: Ostertagiinae)
}

\author{
Abdurakhim Kuchboev ${ }^{1}$, Khanifakhon Sobirova', Rokhatoy Karimova', Oybek Amirov', \\ Georg von Samson-Himmelstjerna ${ }^{2}$ and Jürgen Krücken ${ }^{2^{*}}$
}

\begin{abstract}
Background: The genus Marshallagia (Family Haemonchidae, subfamily Ostertagiinae) contains multiple species of nematodes parasitising the abomasum (or duodenum) of ruminants, in particular of Caprinae. Male specimens have been described to be polymorphic with the frequent/major morphotype initially described in the genus Marshallagia while the minor/rare morphotype was initially often placed in the genus Grossospicularia. Due to common morphological features, certain pairs of morphotypes were suggested to belong to the same species such as Marshallagia marshalli/M. occidentalis. However, molecular evidence to confirm these pairs of morphotypes belonging to the same species is missing.
\end{abstract}

Methods: In the present study, Marshallagia sp. were collected from domestic sheep in Uzbekistan. Male specimens were morphologically described with particular emphasis on the structure of the bursa copulatrix. After DNA isolation from morphologically identified specimens, PCRs targeting the ribosomal internal transcribed spacer 2 (ITS2) and mitochondrial cytochrome c oxidase subunit 1 (cox1) regions were conducted. After Sanger sequencing, maximum likelihood phylogenetic analyses and pairwise identities between sequences were calculated.

Results: The major morphotypes of M. marshalli, M. schumakovitschi and M. uzbekistanica and the minor morphotypes M. occidentalis, M. trifida and M. sogdiana were identified and their morphology was documented in detail. ITS2 sequences showed little variation and did not allow diagnosing species. In contrast, phylogenetic analysis of cox 1 sequences identified highly supported clusters and verified that M. marshalli, M. occidentalis and M. uzbekistanica are different morphotypes of the species M. marshalli while M. schumakovitschi and M. trifida represent distinct morphotypes of M. trifida. For M. sogdiana no corresponding major morphotype could be identified in the present study. Due to a large barcoding gap, comparison of cox 1 sequences in terms of percent identity was sufficient to reliably assign the sequences to a particular species without phylogenetic analysis.

Conclusions: The data presented here create a framework that will allow the classification of other members of the genus in the future and underline that parallel morphological and molecular analysis of specimens is crucial to improve the taxonomy of polymorphic species.

Keywords: Marshallagia, Polymorphic species, Parasitic nematodes, Ruminants, Barcoding gap

\footnotetext{
*Correspondence: juergen.kruecken@fu-berlin.de

${ }^{2}$ Institute for Parasitology and Tropical Veterinary Medicine, Freie Universität Berlin, Robert-von-Ostertag-Str. 7-13, 14163 Berlin, Germany

Full list of author information is available at the end of the article
}

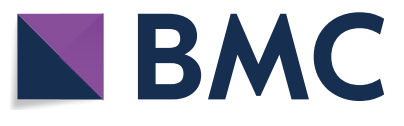

(c) The Author(s) 2020. This article is licensed under a Creative Commons Attribution 4.0 International License, which permits use, sharing, adaptation, distribution and reproduction in any medium or format, as long as you give appropriate credit to the original author(s) and the source, provide a link to the Creative Commons licence, and indicate if changes were made. The images or other third party material in this article are included in the article's Creative Commons licence, unless indicated otherwise in a credit line to the material. If material is not included in the article's Creative Commons licence and your intended use is not permitted by statutory regulation or exceeds the permitted use, you will need to obtain permission directly from the copyright holder. To view a copy of this licence, visit http://creativeco mmons.org/licenses/by/4.0/. The Creative Commons Public Domain Dedication waiver (http://creativecommons.org/publicdomain/ zero/1.0/) applies to the data made available in this article, unless otherwise stated in a credit line to the data. 


\section{Background}

Nematodes of the genus Marshallagia Orloff, 1933 are parasites of the abomasum and duodenum of free-ranging and domesticated ruminants and are most often associated with Caprinae from the Holarctic region $[1,2]$. They have a considerable impact on the metabolism of host animals frequently leading to economic losses [3-5]. According to our data, the species of Marshallagia marshalli Ransom, 1907 is widespread in Uzbekistan and it is the most frequently detected member of this genus [6].

Drozdz $[7,8]$ described that morphological dimorphism is common among males of several species of the subfamily Ostertagiinae, i.e. presence of "major" and "minor" morphotypes in the same host. Major morphotypes are named due to their higher frequency relative to minor morphotypes, not due to differences in size. In contrast to males, females are monomorphic, and this situation has often led to the description of distinct species for these male morphotypes.

In the genus Marshallagia, five dimorphic species have been described by Drozdz [9]. Later, 12 dimorphic species of Marshallagia were differentiated by morphological descriptions incorporating the synlophe in males, the structure of the spicules as well as the genital cone in major and minor morphotype males [2]. In particular, structural characters of the spicules and genital cone were used to distinguish between major and minor morphotypes. The major morphotypes were usually placed in the genus Marshallagia while minor morphotypes were often allocated to Grosspiculagia Orloff, 1933. Thus, major and minor morphotypes of the same species have historically been described as different nominal species, often in separate genera $[9,10]$. However, there are also morphological features that are shared between major and minor morphotypes but can be used to discriminate between species including in particular details of the morphology of esophagus and synlophe as well as the shape of the rays of the copulatory bursa [9]. For instance, M. occidentalis Ransom 1907 (minor morphotype) has clearly distinguishable morphological features discriminating it from $M$. marshalli (major morphotype), but there are clear hints that $M$. marshalli and $M$. occidentalis are synonyms and represent only different morphotypes of $M$. marshalli, whereas $M$. mongolica Shumakovitschi, 1950 appears to be a major morphotype while M. grossospiculum Li, Yin, Kong \& Jang, 1987, which corresponds to Marshallagia sp. 1 of Drozdz [9], is most likely a minor morphotype of the same species. The same relationship presumably also applies to $M$. schumakovitschi Kadyrov, 1959 (major morpotype) and $M$. trifida (Guille, Marotel \& Penisset, 1911) (= Marshallagia sp. 2 of Drozdz [9]) (minor morphotype) [2].
Even less is known about the occurrence of cryptic species in other frequently occurring representatives of the Ostertagiinae, especially in $M$. marshalli due to its presence in several host species $[2,11]$. Regarding nomenclature, taxonomy and phylogeny of the genus Marshallagia, Hoberg et al. [2] proposed that additional studies are required combining morphological and molecular analysis from individual specimens to confirm descriptions of the major species M. skrjabini as well as the minor species $M$. belockani and M. sogdiana. Moreover, it remains an open question as to whether or not M. marshalli/M. occidentalis is distributed in North America and in Eurasia. Marshallagia marshalli/M. occidentalis is the only species of the genus with a Holarctic distribution but detailed comparisons between North American and Central Eurasian populations have not been conducted. Therefore, it should be considered that there are two closely related species with more restricted geographic distribution patterns [2] and that reports of identification of M. marshalli from Eurasian ruminants are due to misidentification.

Based on studies by Drozdz [9], Hoberg et al. [2] and Wyrobisz et al. [12] listed five polymorphic species in the genus Marshallagia: M. marshalli/M. occidentalis; $M$. lichtenfelsi/M. lichtenfelsi f. minor Hoberg Abrams, Pilit \& Jenkins, 2012; M. mongolica/M. grossospiculum; $M$. schumakovitschi/M. trifida; and M. skrjabini/M.belockani. However, to date no consensus on the species composition of the genus Marshallagia has been obtained.

Within the Strongylida, species distinction supported merely by morphological features is difficult, and requires confirmation by means of molecular methods. For instance, recent data have suggested that Cooperia spatulata is just a morphotype of C. punctata [13] while there is evidence that the small strongyle morphospecies Cylicostephanus calicatus and C. minutus are in fact cryptic species complexes of at least two and three genospecies, respectively [14]. The taxonomy of Ostertagiinae is mainly complicated by complex relationship between species and morphotypes in the genus Teladorsagia, but complexity may also be expected among other Ostertagiinae (e.g. in the genera Ostertagia and Marshallagia) [12]. Studies devoted to the problems of taxonomy of strongylid nematodes have shown that noncoding regions of rRNA genes, in ITS1 and particularly ITS2, are well suited to allow taxonomic discrimination and this also applies to members of the Ostertagiinae [15-24]. These studies have led to important insights in the evolution and solved general questions of phylogeny of Ostertagiinae including confirmation of conspecificity for M. marshalli and M. occidentalis [25]. Furthermore, questions regarding conspecificity of supposed major and 
minor morphs and cryptic species within the genus of Orloffia Drozdz, 1965 were solved [26]. There is no unanimous opinion about the taxonomic independence and specific composition of the genus Orloffia. At the same time, there are several species and genera, for which important taxonomic and phylogenetic questions remain unresolved. Additional molecular analyses of ribosomal and mitochondrial DNA will allow understanding these problems in more depth.

Regarding species identification in terms of barcoding properties, mitochondrial DNA sequences were shown to be superior to ribosomal spacers [27]. Recent work on Cooperia spp. (Cooperiidae, Cooperinae) and C. minutus (Strongylidae, Cyathostominae) revealed that combined analyses of mitochondrial and nuclear marker sequences improved species identification and phylogenetic analyses $[13,14]$.

This study aimed to provide sequence data on mitochondrial cytochrome $c$ oxidase subunit 1 ( $\operatorname{cox} 1)$ and nuclear ribosomal intergenic spacer 2 (ITS2) DNA for some species or morphotypes of the genus of Marshallagia. The objective was to morphologically identify individual Marshallagia sp. specimens from Uzbekistan to the species and morphotype level followed by obtaining molecular ITS 2 and cox 1 data from the same specimens in order to clarify the taxonomic status of the local Marshallagia species/morphotypes.

\section{Methods}

\section{Parasite collection and examination}

All parasite material was collected at necropsy from domestic sheep (Ovis aries) from farms in the Kitob district (Kashkadarya region) and in the Shofirkon district (Bukhara region) in Uzbekistan. Mature M. marshalli and M. occidentalis worms were collected from the mucosa of the abomasum of domestic sheep (Ovis aries) in Kashkadarya region (June 2016) while M. schumakovitschi, $M$. trifida, M. sogdiana and M. uzbekistanica were collected from abomasum of sheep in Bukhara region (July 2016). Male specimens were manually cut in two parts: the posterior region was cleared in phenol alcohol $(80 \%$ melted phenol and 20\% ethanol) for examination of the morphological features and the anterior and middle parts were fixed in $70 \%$ ethanol for the molecular studies (Table 1).

\section{Morphological identification}

Marshallagia species were identified according to morphological and morphometrical characters using literature data [1, 2, 9]. All adult male worms isolated from each sheep were morphologically analyzed to identify parasite species. The species identification was established based on caudal bursa according to the features proposed by above literature, especially morphological characters and measurements of spicules, dorsal ray and gubernaculum (Table 1).

An equal mixture of lactic acid and glycerin was used to enlightenment the posterior part of the studied nematodes without additional staining. Also included in the present paper are morphological studies made by the authors analyzing specimens (paratypes or sintypes) of species (M. marshalli Ransom, 1907 and M. schumakovitschi Kadyrov, 1959) in the Central Helminthological Museum FGBNU, Russian Institute of Parasitology, Animal and Plant named after K. I. Skrjabin, Moscow. For this purpose, a microscope ML 2000 equipped with a digital camera (Meiji, Saitama, Japan) was used.

\section{DNA extraction}

For DNA isolation, at least a single specimen of each species/morphotype was used. Before isolation of genomic DNA, the ethanol was removed and the adult nematodes were washed with sterile water and DNA was extracted using the NucleoSpin ${ }^{\circledR}$ Tissue Kit (Macherey-Nagel, Düren, Germany) in accordance with the manufacturer's

Table 1 Specimens of Marshallagia species collected from hosts in Uzbekistan and additional paratype males from the Central Helminthological Museum (Moscow, Russia) for morphological analyses

\begin{tabular}{|c|c|c|c|}
\hline Parasite species & Locality & No. of specimens & Collection \\
\hline Marshallagia marshalli & Kashkadarya district, Uzbekistan & $26 \sigma^{*}$ & CPIZ $10270^{\mathrm{a}}$ \\
\hline M. occidentalis & Kashkadarya district & $220^{*}$ & CPIZ 10278 \\
\hline M. schumakovitschi & Bukhara district, Uzbekistan & $170^{*}$ & CPIZ 10271 \\
\hline M. trifida & Bukhara district & $180^{*}$ & CPIZ 10291 \\
\hline M. sogdiana & Bukhara district & $120^{*}$ & CPIZ 10293 \\
\hline M. uzbekistanica & Bukhara district & 50 & CPIZ 10292 \\
\hline Marshallagia marshalli & Volgograd district, Russia & 20 & CHM $14768^{b}$ \\
\hline M. schumakovitschi & Osh district, Kirgizstan & 20 & CHM 22289 \\
\hline
\end{tabular}

a CPIZ - Collection of the Parasitology Institute of Zoology Uzbekistan Academy of Sciences, Uzbekistan

b CHM - Central Helminthological Museum Russian Institute of Parasitology, Animal and Plant named after K.I. Skrjabin, Russia 
protocol. The DNA was eluted with $50 \mu \mathrm{l}$ elution buffer provided in the kit and stored at $-20{ }^{\circ} \mathrm{C}$ until further use. The extracted DNA was quantified on a Take3 plate in an Epoch plate reader (Biotek, Berlin, Germany).

\section{PCR and cloning}

PCRs were conducted using (i) a combination of the forward and reverse primers flanking the complete ITS2 region [28] and (ii) a partial cox1 gene fragment [29] (Table 2). PCR reactions contained $0.2 \mathrm{mM}$ dNTPs, 250 nM of each primer, 0.4 U Phusion Hot Start II HighFidelity DNA polymerase (Thermo Fisher Scientific, Darmstadt, Germany) and $2 \mu \mathrm{l}$ template DNA in $20 \mu \mathrm{l} 1 \times$ HF buffer. PCRs were performed on a C1000 or S1000 PCR cycler (Bio-Rad, Feldkirchen, Germany). PCR products were purified using DNA Clean \& Concentrator ${ }^{\mathrm{TM}_{-}}-5$ (Zymo Research, Freiburg, Germany) and amplification products were analyzed by agarose gel electrophoresis in 1.0-1.5\% agarose gels. Purified fragments were ligated into the StrataClone Blunt PCR Cloning Vector pSC-Bamp/kan (Agilent, Waldbronn, Germany) and transformed into StrataClone SoloPack competent Escherichia coli cells according to the manufacturer's protocol. Plasmid DNA was purified using the EasyPrep1 Pro kit (Biozym, Hessisch Oldendorf, Germany) and sent for sequencing to LGC Genomics (Berlin, Germany).

\section{Sequence comparisons and phylogenetic analyses}

Sequences from the present study were analyzed together with sequences previously deposited in GenBank. As an outgroup, two sequences per gene from the species Teladorsagia circumcincta, were included. Accession numbers of all sequences from specimens investigated in the present study are provided in Table 3. The ITS 2 and $\operatorname{cox} 1$ sequences were aligned using MAFFT (multiple sequence alignment using fast Fourier transformation) in the Q-INS-I modus that takes predicted RNA secondary structures into account [30] and the M-COFFEE modus of T-Coffee (Treebased Consistency Objective Function for alignment Evaluation) [31], respectively. The cox1 alignment was manually edited to ensure that codons were not interrupted by gaps. For calculation of relative identity (\%)
Table 3 Cytochrome c oxidase subunit 1 (cox1) and second internal transcribed spacer (ITS2) sequences of Marshallagia species from Uzbekistan and the GenBank database from different geographical origins used in this study

\begin{tabular}{llll}
\hline Species & Voucher & \multicolumn{2}{l}{ GenBank ID } \\
\cline { 3 - 4 } & & cox1 & ITS2 \\
\hline Marshallagia marshalli & M2 & MT116991 & MT110920 \\
M. marshalli & M12 & MT116992 & MT110919 \\
M. occidentalis & M14 & MT116997 & MT110967 \\
M. schumakovitschi & M3 & MT116993 & MT110926 \\
M. schumakovitschi & M6 & MT116994 & MT110928 \\
M. schumakovitschi & M8 & MT116995 & MT110929 \\
M. schumakovitschi & M10 & MT116996 & MT110927 \\
M. trifida & M5 & MT116998 & MT118027 \\
M. trifida & M9 & MT116999 & MT118028 \\
M. sogdiana & M4 & MT117000 & MT118024 \\
M. sogdiana & M7 & MT117001 & MT118025 \\
M. sogdiana & M13 & MT117002 & MT118026 \\
M. uzbekistanica & M1 & MT116990 & MT118029 \\
\hline
\end{tabular}

between sequences, alignments were analyzed using the dist.dna function in the ape 4.0 (Analyses of Phylogenetics and Evolution) package [32] in R 4.0.0 statistics software [33]. Identities were calculated as "raw" identities and pairwise deletion of positions with gaps was turned on. Comparisons of sequences within the genus Marshallagia were sorted into the individual intraspecies comparisons and a single category containing all interspecies comparisons. The identity in percent for all these comparisons within the genus Marshallagia were compared using the Kruskall-Wallis test followed by a Conover-Iman post-hoc test with the function posthoc. kruskal.conover.test as implemented in the $\mathrm{R}$ package PMCMR 4.3 [34]. All P-values below 0.05 were considered to be statistically significant. Scatter plots were visualized using GraphPad Prism 5.03 (GraphPad, La Jolla, USA).

Phylogenetic analyses were conducted on a single gene level. First, substitution saturation tests were conducted according to Xia et al. [35] using DAMBE 5 (Data Analysis in Molecular Biology and Evolution) software [36]. DAMBE 5 was also used to split the cox 1 alignment into

Table 2 Primers and PCR conditions used for molecular analyses of nematodes

\begin{tabular}{lllll}
\hline Primer & Sequence $\left(55^{\prime}-3^{\prime}\right)$ & Initial denaturation & Denaturation/annealing/extension & Final elongation \\
\hline NC1 & ACGTCTGGTTCAGGGTTGTT & $98{ }^{\circ} \mathrm{C}$ for $30 \mathrm{~s}$ & $40 \times: 98^{\circ} \mathrm{C}$ for $10 \mathrm{~s} ; 55^{\circ} \mathrm{C}$ for 30 s; $72{ }^{\circ} \mathrm{C}$ for $30 \mathrm{~s}$ & $72^{\circ} \mathrm{C}$ for $10 \mathrm{~min}$ \\
NC2 & TTAGTTCTTTTCCTCCGCT & & \\
COI_Nema_FW & GAAAGTTCTAATCATAARGATATTGG & $95^{\circ} \mathrm{C}$ for 2 min & $35 \times: 95^{\circ} \mathrm{C}$ for $1 \mathrm{~min} ; 48^{\circ} \mathrm{C}$ for 1 min; $72{ }^{\circ} \mathrm{C}$ for 1 min & $72^{\circ} \mathrm{C}$ for 5 min \\
COI_Nema_Rv & ACCTCAGGATGACCAAAAAAYCAA & & & \\
\hline
\end{tabular}


one partition for the first and second and another partition for the third codon position. Maximum likelihood phylogenetic trees were calculated using IQ-TREE [14] on the IQ-TREE server (http://iqtree.cibiv.univie.ac.at). Using the ModelFinder option of IQ-TREE [37], autodetermination of the best model applying the Bayesian information criterion was performed including models with FreeRate heterogeneity. Ultrafast bootstrapping (1000 bootstrapped replicates) [38] and the ShimodairaHasegawa approximate likelihood ratio test (SH-aLRT) (1000 replicates) [39] were used to obtain node support statistics. The command line in IQ-TREE for ITS2 sequences was: iqtree -s infile.fas -st DNA -m TESTNEW -bb 1000 -alrt 1000. For cox 1 sequences, separate models were fitted for codon positions 1 and $2 v s$ codon position 3 using the command line: iqtree -s COI_FcC_infile. fas -spp partition_file.txt -pre infile.fas - m TESTNEW -bb 1000 -alrt 1000. Phylogenetic trees were visualized in FigTree 1.1.4 and further edited in CorelDraw 20.

\section{Results and discussion Morphological identification}

Six morphotypes of Marshallagia species were found within the present material. Major and minor Marshallagia species were isolated from domesticated sheep in Uzbekistan, separated according to their morphological identification (Fig. 1) and morphometric comparison (Table 4) and assigned to M. marshalli, M. schumakovitschi, M. uzbekistanica, M. occidentalis, M. trifida and M. sogdiana. As detailed above, Drozdz [7, 8] described the phenomenon of regular co-occurrence of rather rare (minor) species in pairs with the more numerous (major) species, which, together with subtle morphological features, lead to the hypothesis that they represent different morphotypes of the same species. Based on this hypothesis, the analyzed specimens of Marshallagia were divided into six separate morphotypes that were grouped into three species with co-existing morphotypes (Fig. 1). The original micrographs of the specimens that were used for molecular analysis are presented for one exemplary individual per morphotype: M. marshalli (Fig. 1a); $M$. schumakovitschi (Fig. 1b); M. uzbekistanica (Fig. 1c); $M$. occidentalis (Fig. 1d); M. trifida (Fig. 1e); and M. sogdiana (Fig. 1f). A detailed morphological description of the different morphotypes is given in Additional file 1: Text S1.

The most reliable characters for differentiation among species of Marshallagia, and specifically of the major morphotypes of the respective species, include the placement of the trifurcation of the spicule tips, the form of the dorsal and ventral processes (relative length, curved or straight), and the chitinized structure of the tip of respective processes.

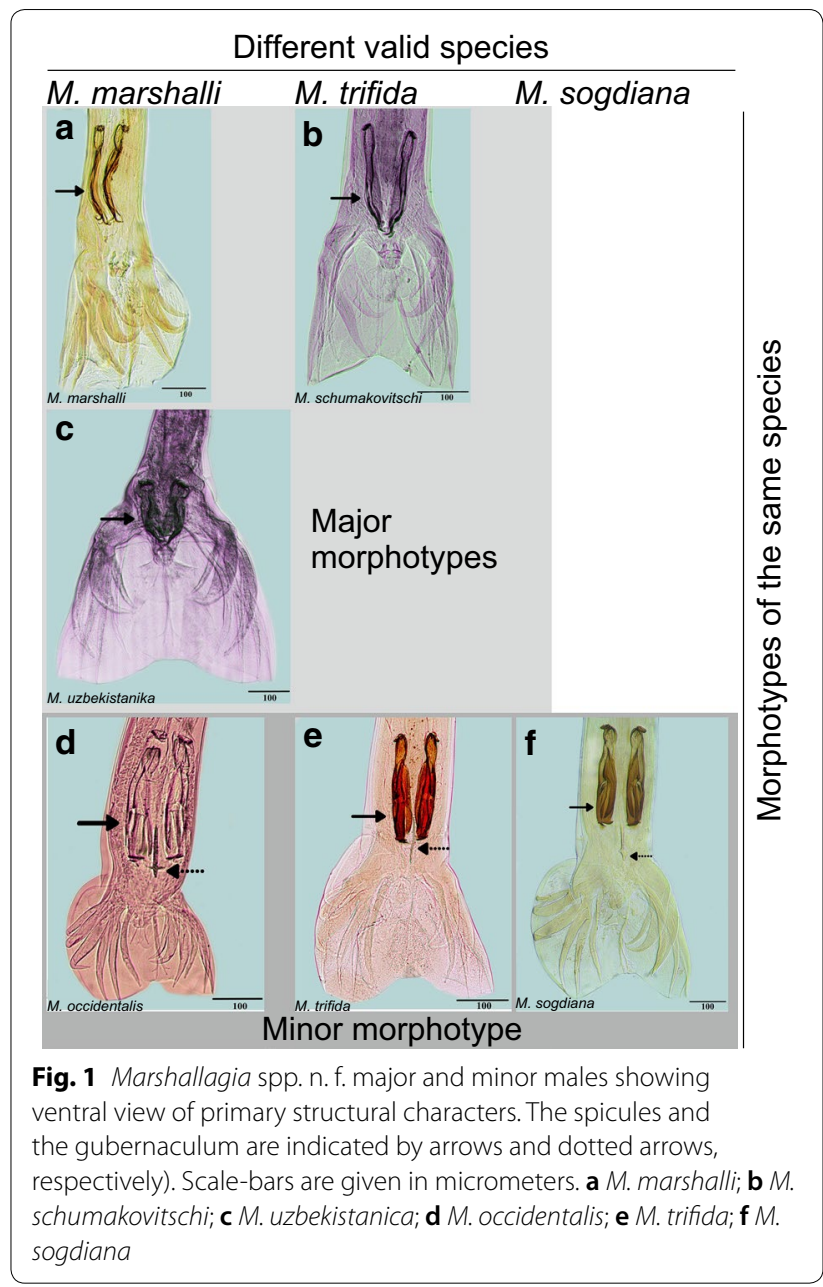

The three major morphotypes designated as $M$. marshalli, M. schumakovitschi and M. uzbekistanica (Fig. 1ac) differ by the distal ends of spicule processes, strongly curved for the first one and with tubercles for the second and third species. All three major morphotypes are characterized by the absence of a gubernaculum (Fig. 1a-c).

Marshallagia marshalli (Ransom, 1907) is the typespecies for the genus. Ransom [40, 41] described this species and the minor morphotype, $M$. occidentalis, based on specimens in domesticated sheep (O. aries L.) from North America. In specimens of the major morphotype of $M$. marshalli the spicules are strongly curved in lateral view, $210-310 \mu \mathrm{m}$ in length, $42-54 \mu \mathrm{m}$ in width; eyelet at trifurcation prominent; with dorsal and ventral process nearly equal in length; gubernaculum absent or strongly chitinized (Fig. 1a). The M. marshalli morphotypes studied by us corresponded to the morphology of the paratype of M. marshalli (CHM 14768) (Table 1).

Marshallagia schumakovitschi differs from M. marshalli in the structure of the spicule. The most distal sixth part of the spicules is divided into three processes. 
Table 4 Morphometric data for the male specimens (major and minor morphotypes) of the morphospecies of Marshallagia in domestic sheep from Uzbekistan, based on new observations during the current study

\begin{tabular}{|c|c|c|c|c|c|c|}
\hline Characters & M. marshalli $(n=26)$ & $\begin{array}{l}\text { M. occidentalis } \\
(n=22)\end{array}$ & $\begin{array}{l}\text { M. schumakovitschi } \\
(n=17)\end{array}$ & M. trifida $(n=18)$ & M. sogdiana $(n=12)$ & $\begin{array}{l}\text { M. uzbekistanica } \\
(n=5)\end{array}$ \\
\hline Body length & $\begin{array}{l}680-1400 \\
\quad(1050 \pm 39)\end{array}$ & $\begin{array}{l}1000-1600 \\
(1330 \pm 51)\end{array}$ & $\begin{array}{l}1140-1380 \\
(1190 \pm 73)\end{array}$ & $\begin{array}{l}830-17000 \\
(1396 \pm 88)\end{array}$ & $\begin{array}{l}940-1850 \\
\quad(1355 \pm 177)\end{array}$ & $\begin{array}{l}734-1586 \\
\quad(1240 \pm 28)\end{array}$ \\
\hline Body width & $125-257(207 \pm 9)$ & $180-260(210 \pm 8)$ & $112-201(175 \pm 4)$ & $131-212(170 \pm 9)$ & $129-235(169 \pm 21)$ & $294-428(340 \pm 8)$ \\
\hline $\begin{array}{l}\text { Diameter of anterior } \\
\text { region }\end{array}$ & $11-26(21 \pm 1)$ & $36-46(41 \pm 1)$ & $21-33(26 \pm 3)$ & $41-62(50 \pm 3)$ & $39-65(47 \pm 4)$ & $17-23(21 \pm 4)$ \\
\hline $\begin{array}{l}\text { Distance from } \\
\text { cervical capsule to } \\
\text { anterior extremity }\end{array}$ & 292-491 (381 \pm 13$)$ & $371-479(414 \pm 8)$ & $391-455(405 \pm 15)$ & $337-581(424 \pm 28)$ & $165-215(188 \pm 9)$ & $401-448(429 \pm 10)$ \\
\hline $\begin{array}{l}\text { Distance from } \\
\text { nerve-ring to } \\
\text { anterior extremity }\end{array}$ & 239-391 (318 \pm 7$)$ & $291-416(350 \pm 10)$ & $220-388(290 \pm 12)$ & $251-411(320 \pm 10)$ & $247-395(295 \pm 12)$ & $151-221(173 \pm 9)$ \\
\hline Esophagus length & $211-319(242 \pm 18)$ & $751-991(890 \pm 20)$ & $781-957(860 \pm 20)$ & $672-932(774 \pm 31)$ & 719-793 (748 \pm 23$)$ & $65-96(78 \pm 9)$ \\
\hline Esophagus width & $26-39(33 \pm 3)$ & $62-93(71 \pm 5)$ & $58-87(65 \pm 4)$ & $59-72(62 \pm 3)$ & $53-68(59 \pm 4)$ & $63-74(68 \pm 4)$ \\
\hline Spicule length & $211-311(250 \pm 10)$ & $218-373(280 \pm 9)$ & $218-301(240 \pm 10)$ & $241-391(295 \pm 9)$ & $254-383(284 \pm 15)$ & $102-147(124 \pm 7)$ \\
\hline Spicule width & $42-54(48 \pm 3)$ & $52-61(57 \pm 2)$ & $53-59(53 \pm 3)$ & $43-57(49 \pm 4)$ & $47-51(48 \pm 5)$ & $38-46(41 \pm 3)$ \\
\hline Dorsal ray length & $321-483(370 \pm 11)$ & $139-317(200 \pm 20)$ & $251-375(300 \pm 10)$ & $231-338(259 \pm 11)$ & $223-315(253 \pm 11)$ & $223-378(241 \pm 11)$ \\
\hline Bursa length & $285-452(360 \pm 8)$ & $653-746(690 \pm 8)$ & $311-415(357 \pm 10)$ & $611-884(709 \pm 12)$ & $593-875(745 \pm 12)$ & $278-548(366 \pm 13)$ \\
\hline
\end{tabular}

Dorsal and ventral processes are nearly equal in length, the ventral process terminates in a simple tip, which may be bent; the dorsal process is weakly sclerotized, blunt and not strongly recurved and extends to near the termination of the main shaft (Fig. 1b).

Marshallagia uzbekistanica is a morphologically atypical form placed in the genus Marshallagia in the original description. Spicules are asymmetric (Fig. 1c). The characteristic of M. uzbekistanica turned out to be a peculiar structure of the spicules, which are weakly chitinized at the proximal end and granular in structure [42].

Minor morphs represented in the present study by the morphotypes $M$. occidentalis, $M$. trifida and $M$. sogdiana correspond to the diagnosis of the genus Grosspiculagia, which is now considered to be a synonym of Marshallagia. In contrast to the major morphs, all minor morphs have thick spicules that are split into three processes. The two more massive processes have cap-shaped distal ends and sometimes a hook-like outgrowth. In contrast to the major morphs, all minor morphs have a transparent, sometimes subtle gubernaculum (Fig. 1d-f).

Marshallagia occidentalis represents the minor morphotype of $M$. marshalli [9]. Near the middle of the spicule length, spicules are divided into three processes: two ventral and one dorsal. A gubernaculum is present but to the rear its diameter is strongly reduced (Fig. 1d).

Marshallagia trifida is the minor morphotype of $M$. schumakovitschi and can be identified based on its spicule structure $[2,9]$. The ventral process of the spicules is strongly curved; the dorsal process extends to the tip of the main shaft of the spicule. The gubernaculum is fusiform (Fig. 1e).

Marshallagia sogdiana is the minor morphotype of $M$. skrjabini Asadov, 1954 and was transferred to that species as a new combination of morphs [2]. The proximal ends of the spicules are characterized by the presence of a peculiar, disc-like structure (Fig. 1f). In the middle of the spicule it is divided into three processes: two ventral and one dorsal. The distal spicules have a membrane in the form of a sheath. A gubernaculum is present.

Thus, based on morphological characters and morphometric comparison of males of Marshallagia sp., two pairs of major and minor morphotypes were identified: M. marshalli/M. occidentalis, M. schumakovitschi/M. trifida while for the pair M. skrjabini/M. sogdiana only the minor morphotype was found (Table 4). In addition, $M$. uzbekistanica was identified as an unusual major morphotype. The data on the morphology of these species from samples collected in Uzbekistan presented here closely corresponds to the previously published data by Asadov [43], Ivashkin [1] and Hoberg et al. [2]. Herein, to scrutinize the identity of the morphs of the genus Marshallagia, molecular studies were conducted in particular to confirm which major and minor morphotypes belong to the same species.

\section{Molecular analyses}

For adult worms collected from domesticated sheep from Uzbekistan and morphologically identified as $M$. marshalli/M. occidentalis, M. schumakovitschi/M. trifida, M.sogdiana and M. uzbekistanica, PCR products for the 
nuclear ITS2 rRNA (321-325 bp excluding the primers) and mitochondrial partial cox1 (696 bp) genes were amplified, cloned and sequenced. All sequences were deposited in GenBank under the accession numbers provided in Table 3.

\section{Internal transcribed spacer 2}

Identity between all ITS2 sequences of the genus Marshalagia from the present study $(n=13)$ or from GenBank revealed between $89.7 \%$ and 100\% identity with $85 \%$ of the pairwise comparisons showing $>95 \%$ identity. A maximum likelihood phylogenetic tree calculated from ITS2 sequences from the present study plus those available in GenBank using two representative T. circumcincta sequences as an outgroup is shown in Additional file 2: Figure S1. The phylogram reveals that the ITS2 sequence contains virtually no phylogenetic signal since (i) there are barely any clusters of sequences showing high statistical support, (ii) sequences assumed to come from different species were virtually identical and (iii) the sequences assigned to the same species are found scattered all over the tree. This indicates that ITS2 sequences are not suitable to address taxonomic or even phylogenetic questions within the genus Marshallagia. This is in agreement with other recent studies analyzing closely related strongyle nematodes showing that ITS2 is an excellent marker to identify the genus, but that closely related species differ only minimally in their ITS 2 sequence and that the phylogenetic signal obtained from ITS2 sequences of Marshallagia specimen was not reliable [13, 14].

\section{Cytochrome $c$ oxidase subunit 1 gene}

The phylogenetic tree calculated from cox 1 sequences from the present study or downloaded from GenBank identified seven highly supported clusters (named I-VII from basal to distal operational taxonomic units (OTUs) in Fig. 2) with very low variability within the clusters. All available Marshallagia sequences come from only three studies. One from China [44] reporting 36 cox 1 sequences, another Chinese study reporting a complete mitochondrial genome annotated as $M$. marshalli [45] and 13 sequences from the present study. The clusters I
(Ostertagia lanceata erroneously assigned to the genus Marshallagia by Lv et al. [44] as indicated by Hoberg et al. [2], IV (M. occidentalis) and V (M. hsui) contained only sequences reported by Lv et al. [44]. In contrast, clusters II ( $M$. sogdiana), III ( $M$. schumakovitschi and $M$. trifida) and V (M. marshalli, M. occidentalis and $M$. uzbekistanica) contained only sequences from the present study. Only in cluster VII (M. mongolica, M. grossospiculum and $M$. marshalli) sequences from two studies are mixed, i.e. $17 \mathrm{M}$. mongolica and two M. grossospiculum sequences published by $\mathrm{Lv}$ et al. [44] and the single complete mitochondrial genome assigned to $M$. marshalli reported by Sun et al. [45].

The fact that two species sequences ( $M$. marshalli and M. occidentalis) both occur in two different clusters clearly indicates that at least some of the published specimens were morphologically misidentified. Neither Lv et al. [44] nor Sun et al. [45] provided any morphological data for the specimens used to obtain their DNA sequences. Lv et al. [44] not even mentioned the criteria used for identification or any species identification key while Sun et al. [45] explicitly stated that morphological identification was difficult, and that molecular identification was used. They report that the ITS sequences obtained from their specimens were $99 \%$ identical to sequences KT428384 and HQ389231 deposited in GenBank as M. marshalli. However, in the ITS2 phylogenetic analysis presented in Additional file 2: Figure S1, these two sequences are (i) not grouped closely together and (ii) also virtually identical to ITS2 sequences deposited in GenBank for other Marshallagia species. Based on the present findings it can be assumed that the complete mitochondrial genome reported by Sun et al. [45] does not represent a $M$. marshalli but instead a $M$. mongolica sequence.

Considering each of the highly supported major clusters in Fig. 2, identification of a valid species can be further confirmed by looking at the barcoding gap. Figure 3 shows raw percent identity for all pairwise comparisons between Marshallagia sp. sequences from the alignment used to calculate the cox 1 phylogenetic tree in Fig. 2. It clearly exhibits that all intraspecies comparisons show

\footnotetext{
(See figure on next page.)

Fig. 2 Maximum likelihood phylogentic tree for Marshallagia species based on cytochrome c oxidase subunit 1 (cox 1) gene. Teladorsagia circumcincta was used as the outgroup while Ostertagia lanceata was included since data are annotated as Marshallagia lanceata in GenBank. Branch support is presented with results of the rapid bootstrap analysis before and of the Shimodaira-Hasegawa approximate likelihood ratio test behind the slash. Seven highly supported clusters (indicated by Roman numbers I-VII from basal to distal) are considered valid species: $O$. lanceatea (I); M. sogdiana (II); M. trifida (III) including the morphotype M. schumakovitschi; the presumably misidentified M. occidentalis from China (IV); M. hsui (V); M. marshalli (VI) including the morphotypes M. occidentalis and M. uzbekistanica from Uzbekistan; and M. mongolica (VII) including the morphotype M. grossospiculum and a presumably misidentified M. marshalli from China. If more than one morphotype is present in a cluster, the valid species name is printed in bold. Numbers after species names show voucher designations from Lv et al. [44] while M1-M14 are voucher designations from the present study. Abbreviations: CN, China; UZ, Uzbekistan; ?, apparently misidentified specimens
} 


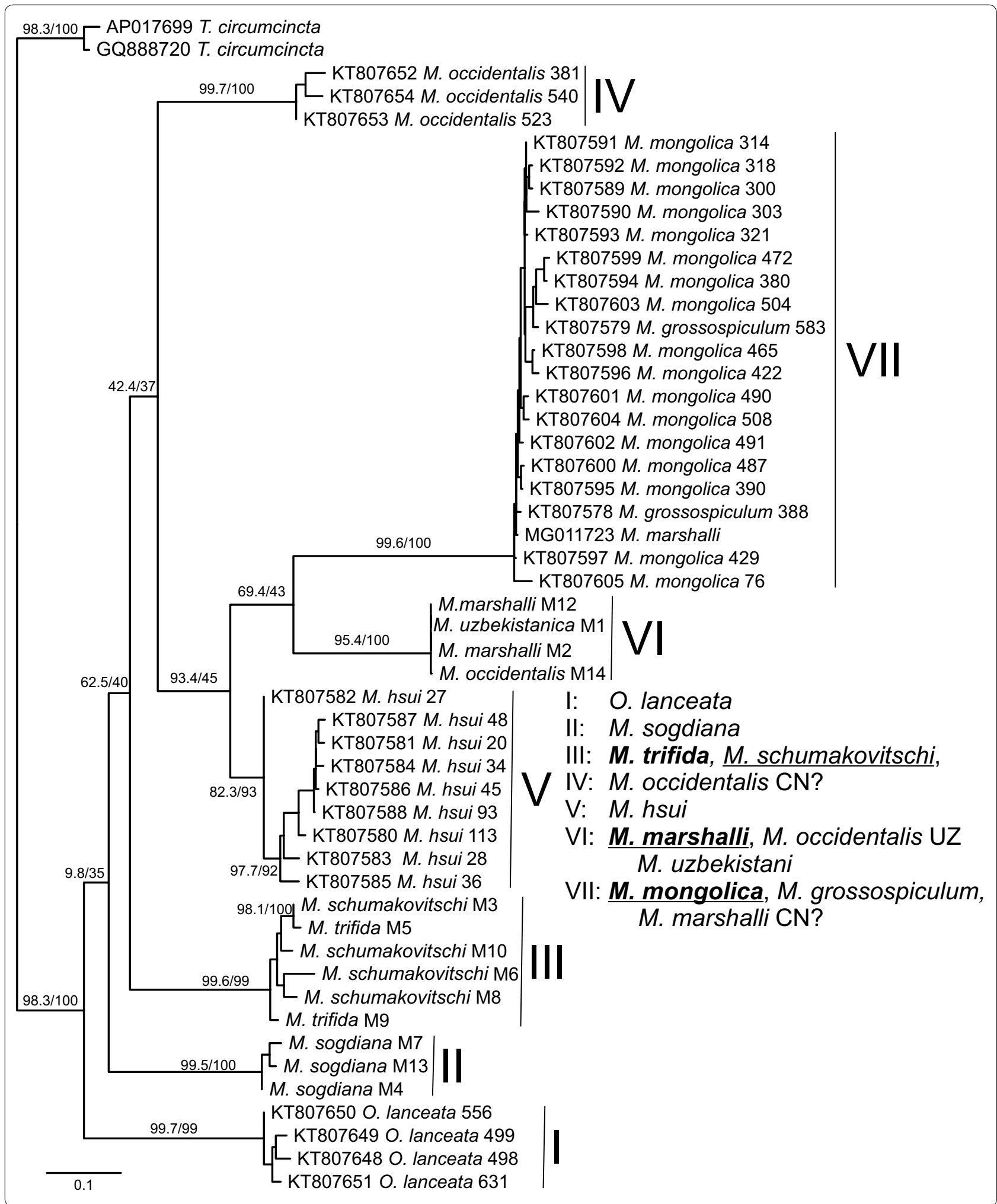




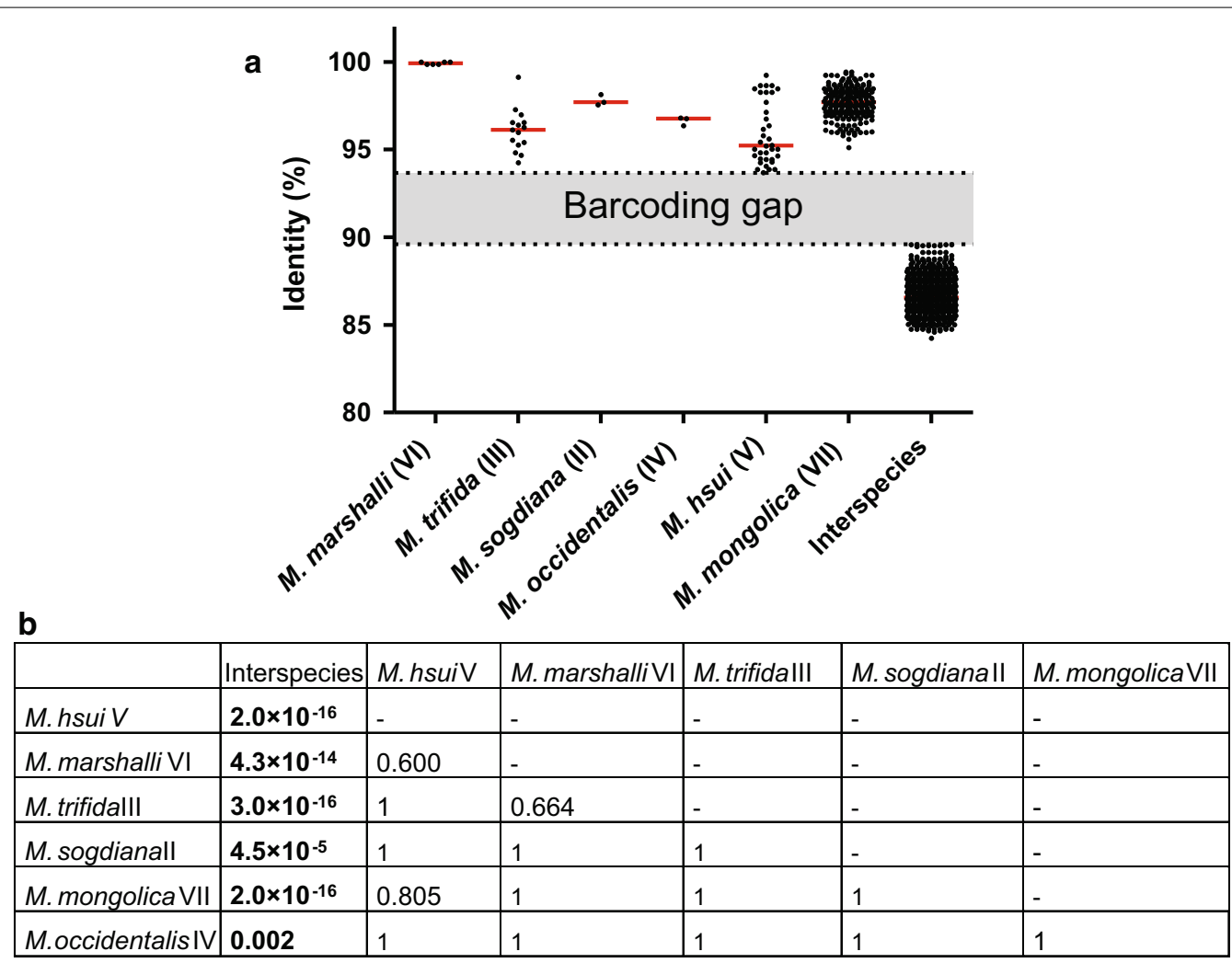

Fig. 3 Sequence identity for cytochrome coxidase subunit 1 (cox1) gene comparisons within and between species. The species Marshallagia sogdiana (II), M. trifida (III) including the morphotype M. schumakovitschi, the presumably misidentified M. occidentalis from China (IV), M. hsui (V), M. marshalli (VI) including the morphotypes M. occidentalis and M. uzbekistanica and M. mongolica including the morphotype M. grossospiculum were included. The different groups (II-VII) correspond to the groups in the phylogenetic tree in Fig. 2. a Scatterplots with medians are shown for all comparisons within these particular species or for any inter-species comparison. The barcoding gap between intra- and interspecies comparisons is indicated by the gray area. Median values for comparisons were significantly different in a Kruskal-Wallis $\mathrm{H}$ test $\left(X^{2}=877.95, d f=266, P<0.001\right)$. b A Conover post-hoc test comparing all different groups revealed that identities of all intra-species comparisons were significantly higher than interspecies comparisons. In contrast, identities of the intra-species comparisons for different species did not show any significant difference

identities between $93.7 \%$ and $100 \%$ while all interspecies comparisons are in the range of $84.2-89.6 \%$. Comparisons between different morphotypes of the same species are in the same range as comparisons between identical morphotypes. Since ranges of intra- and inter- species comparisons are not overlapping (barcoding gap), a simple calculation of identities between sequences will be sufficient to assign a sequence to a particular species allowing diagnosis without phylogenetic reconstruction.

\section{Polymorphic Marshallagia species}

The data presented here clearly show that $M$. marshalli is a polymorphic species and that M. occidentalis and $M$. uzbekistanica should be considered synonyms and that their descriptions detail the morphology of rare morphotypes of $M$. marshalli. In addition, the morphotypes $M$. schumakovitschi and M. trifida belong to the same species. Although M. schumakovitschi represents the major morphotype, this name should be considered a synonym of $M$. trifida since $M$. trifida was described earlier and this name has therefore priority according to the rules of zoological nomenclature. Data from Lv et al. [44] furthermore suggest that $M$. grossospiculum is a minor morphotype of the species $M$. mongolica and only the latter represents a valid species name. However, absence of morphological data in the publication by Lv et al. [44] suggests that further confirmation is warranted.

It remains unclear whether all Marshallagia species are polymorphic but the new data presented here confirm certain pairs of morphotypes that must be considered to be the same species. For other species, the availability of cox1 sequences will allow to group more morphotypes once new sequences from morphologically identified and documented material become available. 


\section{Marshallagia DNA sequences from specimens of unclear origin}

The phylogenetic tree in Fig. 2 contains several GenBank entries from samples of doubtable origin. In particular, there is no available description for $M$. hsui and the name is considered to be a nomen nudum [2]. The sequences obviously belong to a Marshallagia species but without a detailed, publicly available morphological description, it is impossible to decide whether they represent an undescribed species or if they should be assigned to one of the many described species for which no $\operatorname{cox} 1$ reference sequences are currently available.

The M. occidentalis specimens from China for which no morphological description was provided by Lv et al. [44] have presumably been identified incorrectly. The sequences apparently represent Marshallagia sequences but the assignment to $M$. occidentalis is in contrast to all data summarized by Hoberg et al. [2] and to the results of the present study, which all consider M. occidentalis as a minor morphotype of $M$. marshalli. Without voucher material deposited in a museum, the taxonomic value of these sequences will remain very limited and only after identical/highly similar sequences will be reported with a detailed morphological description and deposited voucher material, the sequences will be of epidemiological and taxonomic value.

\section{Conclusions}

Species limits remain poorly defined within the genus Marshallagia (and presumably other, particularly polymorphic Ostertagiinae as well) where subtle morphological differences, high morphological or genetic variability, incomplete descriptions, and circumscribed differential diagnoses hinder identification [2, 43, 46, 47]. It was shown in the present study that at a number of described and named taxa from Eurasia are in fact only synonyms of previously established major or minor morphotypes. For holarctic species such as M. marshalli with its different morphotypes, it would be highly interesting to compare Eurasian and North American specimens regarding mitochondrial genotypes to determine if there is further population genetic structuring or even different genospecies on both continents. In any case, free access to species descriptions, including approaches to overcome language barriers such as very limited accessibility of original parasitic nematode descriptions in Russian for western parasitologists and vice versa, would be required to improve the situation. Missing of accurate and detailed figures and difficult access to representative type-specimens is especially problematic and complicates the possibility of complete and direct comparisons among otherwise similar species and respective morphotypes [2].
Accordingly, comprehensive revision of the genus Marshallagia appears warranted but is currently unfortunately unrealistic.

\section{Supplementary information}

Supplementary information accompanies this paper at https://doi. org/10.1186/s13071-020-04265-1.

Additional file 1: Text S1. Detailed morphological description of the Marshallagia species and morphotypes.

Additional file 2: Figure S1. Maximum likelihood phylogenetic tree calculated based on ITS2 sequences. Teladorsagia circumcincta sequences were used as the outgroup. Rapid bootstrapping and ShimodairaHasegawa approximate likelihood ratio test results are shown before and after the slash, respectively. M1-M14 indicate voucher designations from the present study.

\section{Abbreviations}

rRNA: ribosomal RNA; ITS1: internal transcribed spacer 1; ITS2: internal transcribed spacer 2; cox1: cytochrome coxidase subunit 1 gene.

\section{Acknowledgments}

The authors thank Sabrina Ramünke for constant assistance provided regarding molecular studies during the whole period of work.

\section{Authors' contributions}

AK conceived and designed the study and prepared the first draft of the manuscript. OA and RK collected specimens for the study. KS carried out morphometric analysis of species. AK and OA performed the morphological description. AK carried out the molecular work and JK completed the bioinformatical and statistical analysis. JK and GVS oversaw the study and manuscript preparation. All authors read and approved the final manuscript.

\section{Funding}

Open access funding provided by Projekt DEAL. This study has been carried out within the framework of the DEUTSCHER AKADEMISCHER AUSTAUSCHDIENST (DAAD) — funding program 57313677, Bilateral Exchange of Academics, 2017, that supported the visit of AK to the Institute of Parasitology and Tropical Veterinary Medicine, Freie Universität Berlin, Germany as a host, and part of the study was supported by project number FA-A8-T004, Institute of Zoology, Uzbekistan Academy of Sciences.

\section{Availability of data and materials}

All data generated or analyzed during this study are included in this article and its additional files. Sequence data were deposited in the GenBank database under the accession numbers listed in Table 3.

\section{Ethics approval and consent to participate}

The parasite samples used in the study were collected from sheep, when the animals were slaughtered for meat and we obtained the specimens from the abomasa and intestines with the permission of the farm owners.

\section{Consent for publication}

Not applicable.

\section{Competing interests}

The authors declare that they have no competing interests.

\section{Author details}

${ }^{1}$ Institute of Zoology, Uzbekistan Academy of Sciences, Bogishamol str. 232B, Tashkent 100053, Uzbekistan. ${ }^{2}$ Institute for Parasitology and Tropical Veterinary Medicine, Freie Universität Berlin, Robert-von-Ostertag-Str. 7-13, 14163 Berlin, Germany.

Received: 28 March 2020 Accepted: 29 July 2020

Published online: 12 August 2020 


\section{References}

1. Ivashkin VM, Oripov AO, Sonin MD. Manual for determinative helminths of sheep and goats. Moscow: Nauka; 1989.

2. Hoberg EH, Abrams A, Pilitt PA, Jenkins EJ. Discovery and description of a new Trichostrongyloid species (Nematoda: Ostertagiinae), abomasal parasites in mountain goat, Oreamnos americanus, from the western Cordillera of North America. J Parasitol. 2012:98:817-46.

3. Irgashev $\mathrm{IH}$. Helminths and helminthiasis karakul sheep. Tashkent: Fan Publisher; 1972

4. Oripov SA. Trihostrongylidiasis of sheep in Uzbekistan and their control. Ph.D. thesis, All-Union Scientific Research Institute for Parasitology K. I. Skryabin, Russia; 1983.

5. Abramatov MB, Amirov OO, Ruziev BKh, Kuchboev AE. Helmintocenosis of abomasum from domestic ruminants of Uzbekistan. Biol Sci Kazakhstan. 2014;1:28-37.

6. Kuchboev AE, Amirov OO, Karimova RR, Asakawa M. Nematodes in the digestive tract of domestic ruminants in Uzbekistan. Jpn J Vet Parasitol. 2016;15:124-9.

7. Drozdz J. The question of genetic isolation and of permanent coincidence of some species of the subfamily Ostertagiinae. In: Proceedings of the third international congress of parasitology. Munich; 1974. p. 477-8.

8. Drozdz J. Genetic isolation as a criterion for defining a species within the parasitic nematodes. Wiad Parazytol. 1979;25:171-83.

9. Drozdz J. Polymorphism in the Ostertagiinae Lopez-Neyra, 1947 and comments on the systematics of these nematodes. Syst Parasitol. 1995;32:91-9

10. Gibbons LM, Khalil LF. A key for the identification of genera of the nematode family Trichostrongylidae Leiper, 1912. J Helminthol. 1982:56:185-233.

11. Lichtenfels JR, Pilitt A. Cuticular ridge patterns of Marshallagia marshalli and Ostertagia occidentalis (Nematoda: Trichostrongyloidea) parasitic in ruminants of North America. Proc Helminthol Soc Wash. 1989;56:173-82.

12. Wyrobisz A, Kowal J, Nosal P. Insight into species diversity of the Trichostrongylidae Leiper, 1912 (Nematoda: Strongylida) in ruminants. J Helminthol. 2016:90:639-64.

13. Ramünke $S$, de Almeida Borges F, von Son-de Fernex E, von SamsonHimmelstjerna G, Krücken J. Molecular marker sequences of cattle Cooperia species identify Cooperia spatulata as a morphotype of Cooperia punctata. PLoS One. 2018;13:e0200390.

14. Bredtmann CM, Krücken J, Kuzmina T, Louro M, de Madeira Carvalho LM, von Samson-Himmelstjerna G. Nuclear and mitochondrial marker sequences reveal close relationship between Coronocyclus coronatus and a potential Cylicostephanus calicatus cryptic species complex. Infect Genet Evol. 2019;75:103956

15. Zarlenga DS, Hoberg EP, Stringfellow F, Lichtenfels JR. Comparisons of two polymorphic species of Ostertagia and phylogenetic relationships within the Ostertagiinae (Nematoda:Trichostrongyloidea) inferred from ribosomal DNA repeat and mitochondrial DNA sequences. J Parasitol. 1998:84:806-12.

16. Dallas JF, Irvine RJ, Halvorsen O. DNA evidence that Ostertagia gruehneri and Ostertagia arctica (Nematoda: Ostertagiinae) in reindeer from Norway and Svalbard are conspecific. Int J Parasitol. 2000;30:655-8.

17. Chilton NB, Newton LA, Beveridge I, Gasser RB. Evolutionary relationships of trichostrongyloid nematodes (Strongylida) inferred from ribosomal DNA sequence data. Mol Phylogenet Evol. 2001;19:367-86.

18. Santin-Duran M, de la Fuente C, Alunda JM, Rosental BM, Hoberg EP. Identical ITS-1 and ITS-2 sequences suggest Spiculopteragia asymmetrica and Spiculopteragia quadrispiculata (Nematoda: Trichostrongylidae) constitute morphologically distinct variants of a single species. J Parasitol. 2002:88:417-8

19. Kuznetsov DN. The results of the comparative study of the spacer region of the ribosomal DNA Teladorsagia circumcincta and T. trifurcata (Nematoda: Ostertagiinae). Russian Parasitol J. 2009;2:16-23.

20. Kuznetsov DN. On the question of species identity Ostertagia ostertagi and Ostertagia lyrata (Nematoda: Ostertagiinae). Parazitologiya. 2010:44:226-31.

21. Aksenov AP. Nematode's subfamily Ostertagiinae Lopez-Neyra, 1947: systematics and phylogeny. Ph.D. Thesis, A. N. Severtsov Institute of Ecology and Evoltion, Russia; 2013.

22. Amirov OO, Kuchboev AE. Molecular characterization of nematodes Teladorsagia circumcincta and T. trifurcata (Trichostrongylidae: Ostertagiinae) using the spacer regions of ribosomal DNA. Bull Natl Univ Uzbekistan. 2013;4:278-84

23. Amirov OO, Kuchboev AE. Molecular genetic analysis of Ostertagia ostertagi and O. Iyrata (Trichostrongylidae). Bull Gullistan State Univ. 2014:1:28-32.

24. Amirov OO, Mirzaeva GC, Kuchboev AE. Molecular genetic analysis species of Teladorsagia sircumcincta and Teladorsagia davtiani (Nematoda: Ostertagiinae). Infect Immunol Pharmacol. 2014;4:25-30.

25. Dallas JF, Irvine RJ, Halvorsen O. DNA evidence that Marshallagia marshalli Ransom, 1907 and M. occidentalis Ransom, 1907 (Nematoda: Ostertagiinae) from Svalbard reindeer are conspecific. Int J Parasitol. 2001;2001(50):101-3.

26. Kuznetsov DN. Taxonomic revision of the genus Orloffia (Nematoda: Ostertagiinae) based on an ITS-2 rDNA study. Biol Bull. 2001;38:608-14.

27. Blouin MS. Molecular prospecting for cryptic species of nematodes: mitochondrial DNA versus internal transcribed spacer. Int J Parasitol. 2002;32:527-31.

28. Gasser RB, Chilton NB, Hoste H, Beveridgi L. Rapid sequencing of rDNA from single worms and eggs of parasitic helminthes. Nucleic Acids Res. 1993:21:2525-6.

29. Duscher GG, Harl J, Fuehrer HP. Evidence of Troglotrema acutum and Skrjabingylus sp. coinfection in a polecat from lower Austria. Helminthologia. 2015:52:63-6.

30. Katoh K, Standley DM. MAFFT multiple sequence alignment software version 7: improvements in performance and usability. Mol Biol Evol. 2013;30:772-80.

31. Taly JF, Magis C, Bussotti G, Chang JM, Di Tommaso P, Erb I, et al. Using the T-Coffee package to build multiple sequence alignments of protein, RNA, DNA sequences and 3D structures. Nat Protoc. 2011;6:1669-82.

32. Popescu AA, Huber KT, Paradis E. ape 3.0: new tools for distancebased phylogenetic and evolutionary analysis in R. Bioinformatics. 2012;28:1536-7.

33. R Development Core Team. R: A language and environment for statistical computing. R Foundation for Statistical Computing. Vienna: R Foundation for Statistical Computing; 2020. https://www.R-project.org.

34. Pohlert T. The pairwise multiple comparison of mean ranks package (PMCMR). R package. 2014. http://CRAN.R-project.org/package=PMCMR.

35. Xia X, Lemey P. editors. 209. Assessing substitution saturation with DAMBE. In: Lemey P, Salemi M., Vandamme A-M, editors. The Phylogenetic Handbook: A Practical Approach to Phylogenetic Analysis and Hypothesis Testing. 2nd ed. Cambridge: Cambridge University Press; 2009. p. 615-30.

36. Xia X. DAMBE5: a comprehensive software package for data analysis in molecular biology and evolution. Mol Biol Evol. 2013;30:1720-8.

37. Kalyaanamoorthy S, Minh BQ, Wong TKF, von Haeseler A, Jermiin LS. ModelFinder: fast model selection for accurate phylogenetic estimates. Nat Methods. 2017;14:587.

38. Hoang DT, Vinh LS, Chernomor O, Minh BQ, von Haeseler A. UFBoot2: improving the ultrafast bootstrap approximation. Mol Biol Evol. 2017:35:518-22.

39. Guindon S, Dufayard JF, Lefort V, Anisimova M, Hordijk W, Gascuel O. New algorithms and methods to estimate maximum-likelihood phylogenies: assessing the performance of PhyML 3.0. Syst Biol. 2010;59:307-21.

40. Ransom BH. Notes on parasitic nematodes, including descriptions of new genera and species, and observations of life histories. U.S. Department of Agriculture, Bureau of Animal Industry Circular 116; 1907.

41. Ransom BH. The nematodes parasitic in the alimentary tract of cattle, sheep and other ruminants. Bureau of Animal Industry, U.S. Department of Agriculture, U.S. Government Printing Office, Washington, D.C., Bulletin $127 ; 1911$.

42. Azimov DA, Dadaev D. Marshallagia uzbekistanica sp. n., a new nematode of sheep and goats. Uzbek Biol J. 2001;3:52-3.

43. Asadov SM. Helminthofauna ruminants USSR and its ecological and geographical analysis. Baku: Academy of Sciences of AzSSR; 1960

44. Lv J, Zhang Y, Feng C, Yuan X, Sun D, Deng J, et al. Species discrimination in the subfamily Ostertagiinae of northern China: assessment of DNA barcode in a taxonomically challenging group. Parasitol Res. 2016;115:987-96.

45. Sun MM, Han L, Zhang FK, Zhou DH, Wang SQ, Ma J, et al. Characterization of the complete mitochondrial genome of Marshallagia marshalli and phylogenetic implications for the superfamily Trichostrongyloidea. Parasitol Res. 2018;117:307-13. 
46. Hu J, Jiang Y. Marshallagia brevicauda new species (Nematoda, Trichostrongylidae) from sheep. Acta Veterinaria et Zootechnica Sin. 1984;15:257-64.

47. Luo JZ, Zhang HJ, Wu BS, Bai ZY. Two new species of Marshallagia from Qinghai, China. Acta Zootaxonomica Sin. 1993;18:142-8.

\section{Publisher's Note}

Springer Nature remains neutral with regard to jurisdictional claims in published maps and institutional affiliations.
Ready to submit your research? Choose BMC and benefit from:

- fast, convenient online submission

- thorough peer review by experienced researchers in your field

- rapid publication on acceptance

- support for research data, including large and complex data types

- gold Open Access which fosters wider collaboration and increased citations

- maximum visibility for your research: over 100M website views per year

At BMC, research is always in progress.

Learn more biomedcentral.com/submissions 